\title{
Long-range memory in internal and forced dynamics of millennium-long climate model simulations
}

\author{
L. Østvand ${ }^{1}$, T. Nilsen ${ }^{2}$, K. Rypdal ${ }^{2}$, D. Divine ${ }^{3}$, and M. Rypdal ${ }^{2}$ \\ ${ }^{1}$ Department of Physics and Technology, UiT The Arctic University of Norway, Troms $\emptyset$, Norway \\ ${ }^{2}$ Department of Mathematics and Statistics, UiT The Arctic University of Norway, Troms $\varnothing$, Norway \\ ${ }^{3}$ Norwegian Polar Institute, Troms $\varnothing$, Norway \\ Correspondence to: L. Østvand (lene.ostvand@uit.no)
}

Received: 26 February 2014 - Published in Earth Syst. Dynam. Discuss.: 25 March 2014

Revised: 19 June 2014 - Accepted: 24 July 2014 - Published: 27 August 2014

\begin{abstract}
Northern Hemisphere $(\mathrm{NH})$ temperature records from a palaeoclimate reconstruction and a number of millennium-long climate model experiments are investigated for long-range memory (LRM). The models are two Earth system models and two atmosphere-ocean general circulation models. The periodogram, detrended fluctuation analysis and wavelet variance analysis are applied to examine scaling properties and to estimate a scaling exponent of the temperature records. A simple linear model for the climate response to external forcing is also applied to the reconstruction and the forced climate model runs, and then compared to unforced control runs to extract the LRM generated by internal dynamics of the climate system. The climate models show strong persistent scaling with power spectral densities of the form $S(f) \sim f^{-\beta}$ with $0.8<\beta<1$ on timescales from years to several centuries. This is somewhat stronger persistence than found in the reconstruction $(\beta \approx 0.7)$. We find no indication that LRM found in these model runs is induced by external forcing, which suggests that LRM on sub-decadal to century time scales in NH mean temperatures is a property of the internal dynamics of the climate system. Reconstructed and instrumental sea surface temperature records for a local site, Reykjanes Ridge, are also studied, showing that strong persistence is found also for local ocean temperature.
\end{abstract}

\section{Introduction}

The presence of long-range memory (LRM) in climatic records is well documented in the geophysics literature. LRM is characterized by an algebraically decaying autocorrelation function (ACF) $\lim _{t \rightarrow \infty} C(t) \propto t^{-\gamma}$ such that $\int_{0}^{\infty} C(t) \mathrm{d} t=\infty$, i.e. $0<\gamma \leq 1$. Equivalently, the power spectral density (PSD) of LRM time series follows a power law $\lim _{f \rightarrow 0} S(f) \propto f^{-\beta}$, where $\beta=1-\gamma$ and $0<\beta<1$. A typical model for an LRM stochastic process is the persistent fractional Gaussian noise (fGn). This is a stationary process with $0<\beta<1$. The cumulative integral (or sum) of such a process has the PSD of the form $S(f) \sim f^{-\beta}$, but with $\beta \rightarrow \beta+2$. Such a process with $1<\beta<3$ is a non-stationary LRM process called a fractional Brownian motion (fBm).

In short time series the ACF estimator is biased and noisy, which makes it difficult to use it for estimating properties of a power-law tail. The reason for the bias is that the sample mean will have to be used, rather than the true mean, in the estimation of the ACF (Lennartz and Bunde, 2009b; Rypdal et al., 2013). In this paper we use the periodogram to estimate the PSD as the first crude characterization of the data, and employ the periodogram, detrended fluctuation analysis (DFA) and wavelet variance analysis (WVA) for parameter estimation.

Most of the LRM studies of climatic time series investigate local time records (e.g. Pelletier, 1997; Weber and Talkner, 2001; Eichner et al., 2003), but LRM has also been found in global observed temperature records (Lennartz and Bunde, 2009a) and reconstructed temperature records for the Northern Hemisphere (Rybski et al., 2006; Mills, 2007). Some surface temperature records from atmosphere-ocean general circulation model (AOGCM) climate models have been analysed with the main result that LRM is not reproduced in agreement with that of observational temperature 
(Syroka and Toumi, 2001; Bunde et al., 2001; Govindan et al., 2001, 2002; Bunde and Havlin, 2002). Some of the model experiments produce temperature with multiple scaling regimes, and some of them yield smaller scaling exponents than the observational temperature. However, in Syroka and Toumi (2001), Bunde et al. (2001), and Govindan et al. (2001) the model experiments all had greenhouse gas forcing as the only dynamic forcing, while remaining external forcings, such as total solar irradiance and volcanic effects, were kept constant. Govindan et al. (2002) and Bunde and Havlin (2002) used experiments (i) where all forcings were fixed, (ii) with fixed forcings except greenhouse gas forcing, and (iii) with fixed forcings except greenhouse gas plus aerosol forcing. Their main conclusion was that the temperature from the model experiments fails to reproduce the scaling behaviour found in observational data, and that the models display large differences in scaling from different sites. Of these scenarios, the one with dynamic greenhouse gas plus aerosol forcing performed better with respect to producing the scaling observed in instrumental temperature records. Global fields of observed and simulated surface temperatures from an AOGCM climate model experiment were studied in Fraedrich and Blender (2003). The experiment was run with fixed forcings. The result from observational data was mostly in agreement with previous studies of temperature in oceanic and coastal regions, but the authors found white noise scaling $(\beta \approx 0)$ at continental interiors. Analysis of a 1000-year temperature simulation from the model experiment produced similar scaling exponents to what was found for the observational data in this study. Blender and Fraedrich (2003) made a similar analysis of temperature from two different model experiments with dynamic greenhouse gas forcing, giving results in agreement with Fraedrich and Blender (2003).

Temperature from model experiments with constant forcings, and time-varying greenhouse gas, sulfate aerosol, ozone, solar, volcanic forcing and various combinations was studied in Vyushin et al. (2004). Scaling exponents for temperature at 16 land sites and 16 sites in the Atlantic Ocean were estimated. They found that inclusion of volcanic forcing considerably improved the scaling behaviour. Rybski et al. (2008) used model experiments with all constant forcing and with dynamic solar, volcanic and greenhouse gas forcing. They analysed data from grid cells all over the globe, but did not investigate global or hemispheric means. They found that for the forced run experiment the temperature showed a scaling exponent in agreement with observational temperature, while the temperature from the control run showed generally lower persistence.

Studies of LRM in temperature records from climate model experiments mostly use temperature from local sites, and some also use temperature spatially averaged over larger regions. Global mean temperature was studied by Syroka and Toumi (2001), but hemispheric means have not been studied with regard to LRM. For observational and reconstructed temperature, global and hemispheric means are also far less studied than local data.

In the present study we analyse scaling properties of surface temperature for the Northern Hemisphere $(\mathrm{NH})$ from palaeoclimate simulations and compare to those of temperature reconstruction by Moberg et al. (2005) which spans the last two millennia. Hemispheric temperature records from four different Earth system climate models are analysed, and both forced runs and control runs are investigated. In order to avoid effects of anthropogenic forcing only data up to the year 1750 are used. This will give an idea of what role other natural external forcing like solar, $\mathrm{CO}_{2}$, volcanic and aerosol forcing play in producing LRM, and indicate whether LRM can arise from internal dynamics alone.

Separation of the LRM arising from internal dynamics from the LRM induced by external forcing can also be achieved from reconstructed and simulated temperature data if the forcing data are known. The method makes use of a simple linear model for the global temperature response (Rypdal and Rypdal, 2013). The response to the external forcing can then be computed and subtracted from the observed or modelled temperature record to yield a residual which represents the internal variability of the climate system. Analysis of this residual and temperature from forced runs and control runs are compared for those models where temperatures from both forced runs and control runs are available.

This paper is organized as follows: Sect. 2 describes the DFA and WVA methods and the response model. Information about the models and the data used can be found in Sect. 3, and the results from the analysis are presented in Sect. 4. Discussion and conclusion follow in Sect. 5.

\section{Methods}

\subsection{Estimation of power spectral density}

The periodogram for the evenly sampled time series $x(1), x(2), \ldots, x(n)$ is defined in terms of the discrete Fourier transform $H_{m}$ as

$S_{m}=\frac{2\left|H_{m}\right|^{2}}{N}, m=1,2, \ldots, N / 2$.

Since our time unit here is the sampling time, the frequency measured in cycles per time unit is $f_{m}=m / N$. The smallest frequency which can be represented in the spectrum, and the frequency resolution, is $1 / N$, and the highest frequency that can be resolved (the Nyquist frequency) is $f_{N / 2}=1 / 2$.

The periodogram has been heavily criticized as an estimator for PSD for a number of reasons, but mainly because of its poor signal-to-noise ratio. Standard error for a single frequency is of the order of the mean, and it will not be improved by a longer time series, because the number of frequencies to which one can distribute the power increases proportionally to $N$. This is a problem if we are interested 
in estimating the power in spectral peaks that are resolved by the frequency resolution $1 / N$, but here we are concerned with the overall shape of the spectrum. Windowing applied to numerical realizations of fGns before computing the periodogram has a weak tendency to suppress the lowest frequencies in the spectrum and hence destroy the scaling. For this reason we show the log-binned periodogram in log-log plots to give an intuitive feeling for the power-law and LRM character $(\beta>0)$ of the spectra.

When estimating the spectral index from the periodogram, one needs to keep in mind that the points will appear with increasing density towards high frequencies in $\log -\log$ plots and hence, if there is imperfect scaling in the form of different slopes of the log-log curve in different parts of the spectrum, such a fit will emphasize the higher end. In dealing with LRM, however, we are mainly concerned with the lower end of the spectrum, so a better approach would be to compute the power in frequency bins which appear with constant width on a logarithmic scale. Straight lines fitted to frequency-binned power spectra will give a better representation of the overall scaling properties of the signal. The same idea applies to all the other techniques that will be described in the following. These represent the scaling in terms of timescale $\tau$, rather than frequency, but when fitting is done in $\log -\log$ plots we fit to curves where the scales are represented by points with constant separation on the log scale.

\subsection{Detrended fluctuation analysis}

The detrended fluctuation analysis (DFA) (Peng et al., 1994; Kantelhardt et al., 2001) was explicitly designed to remove polynomial trends in the data. The method can be summarized in four steps. First, we construct the cumulative sum (the "profile") of the temperature time series $x(t), Y(i)=$ $\sum_{t=1}^{i} x(t)-\langle x\rangle$, where $\langle x\rangle$ denotes the mean. In the second step the profile is divided into $N_{\tau}=N / \tau$ non-overlapping segments of equal length $\tau$. This is done both starting at the beginning and at the end of the profile, so $2 N_{\tau}$ segments are obtained altogether. In the third step, an $n$ th-order polynomial is fitted to, and then subtracted from, each segment. Thus, at this stage we have formed the detrended profile $Y_{\tau}(i)=Y(i)-p_{v}(i)$, where $p_{\nu}(i)$ is the polynomial fitted to the $v$ th segment. In the final step, the variance of each segment, $F^{2}(v, \tau)=\frac{1}{\tau} \sum_{i=1}^{\tau} Y_{\tau}^{2}[(v-1) \tau+i]$, is computed. The fluctuation function is given by the square root of the average over all the segments,

$F(\tau)=\left[\frac{1}{2 N_{\tau}} \sum_{\nu=1}^{2 N_{\tau}} F^{2}(\nu, \tau)\right]^{\frac{1}{2}}$.

The scaling parameter $\beta$ is found through the relation

$F(\tau) \propto \tau^{(\beta+1) / 2}$.

What we have described is the $n$ th-order detrended fluctuation analysis, denoted DFA $n$. It has the property of eliminat- ing the effect of an $(n-1)$ th-order polynomial trend. In this paper we employ second-order DFA, denoted DFA2, which eliminates linear trends. DFA estimates of $\beta$ has also been shown to be rather weakly affected by oscillatory trends, provided the oscillation period is well inside the range of scales investigated (Hu et al., 2001).

\subsection{Wavelet variance analysis}

The continuous wavelet transform is the convolution between a time series $x(t)$ and the rescaled wavelet $\Psi(t / \tau)$ :

$W(t, \tau ; x(t), \Psi(t))=\int_{-\infty}^{\infty} x\left(t^{\prime}\right) \frac{1}{\sqrt{\tau}} \Psi\left(\frac{t^{\prime}-t}{\tau}\right) \mathrm{d} t^{\prime}$.

The mother wavelet $\Psi(t)$ and all rescaled versions of it must fulfil the criterion $\int_{-\infty}^{\infty} \Psi\left(t^{\prime}\right) \mathrm{d} t^{\prime}=0$. For LRM time series, the variance $V_{W}(\tau)=(1 / N) \sum_{t=1}^{N} W^{2}(t, \tau)$ scales as a power law (Flandrin, 1992; Malamud and Turcotte, 1999),

$V_{W}(\tau) \sim \tau^{\beta}$

The method is therefore known as wavelet variance analysis (WVA). In this study we have used the Mexican hat wavelet, which is capable of eliminating linear trends, and denote the method WVA2. The properties of the WVA2 analysis are similar to the DFA2 in that it usually yields similar values of $\beta$. It is, however, much more sensitive to the presence of additional oscillations in the data, which show up as wavy structures in the function $V_{W}(\tau)$. We use it in this paper mainly as a tool (in addition to the periodogram) to detect such oscillations.

\subsection{The response model residual analysis}

For the preindustrial period the most important contributions to the external radiative forcing $F(t)$ are orbital, solar variability and aerosols from volcanic eruptions. Orbital forcing can be computed with high accuracy, and total solar irradiation has been reconstructed for the last 10 millennia. Existing reliable reconstructions of volcanic forcing cover the last millennium. The forcing data used here are further described in Sect. 3. The evolution of the global mean surface temperature anomaly $T$ on decadal to millennial timescales can tentatively be modelled as a linear response to $F(t)$ in addition to a response to stochastic forcing from unresolved spatiotemporal "turbulence" (e.g. forcing of the sea surface temperature from atmospheric weather systems). A simple stochastic-dynamic model (SDM) with an LRM response function is (Rypdal and Rypdal, 2013)

$$
T(t)=\mu[\underbrace{\int_{0}^{t}(t-s)^{\beta / 2-1} F(s) \mathrm{d} s}_{\text {deterministic solution }}+\underbrace{\sigma \int_{0}^{t}(t-s)^{\beta / 2-1} \mathrm{~d} B(s)}_{1 / f^{\beta} \text { noise }}] .
$$


Here $B(s)$ is the Wiener stochastic process whose increment $\mathrm{d} B(s)$ is a Gaussian white noise process and $\sigma \mathrm{d} B(s)$ represents the stochastic component of the forcing. $T(t)$ is the temperature relative to the temperature $T_{0}$ at time $t=0$ (the beginning of the record) and $F=\tilde{F}+F_{0}$ is perturbed forcing $\tilde{F}$ relative to that of a radiative equilibrium at surface temperature $T_{0}$ plus the actual radiative imbalance $F_{0}$ at $t=0$. By definition $\tilde{F}(0)=0 . F_{0}$ is a model parameter which is estimated from the data along with the other model parameters $\beta, \mu$ and $\sigma$. The stochastic part of this solution (the term to the right) has a power spectral density of the form $S(f) \sim f^{-\beta}$, and is fractional Gaussian noise (a stationary process) if $\beta<1$ and a fractional Brownian motion (nonstationary) if $1<\beta<3$.

Estimates of past changes in global climate forcing and its various components exist for the instrumental period as well as for the last millennium. This information can be used in conjunction with the observed temperature records to perform maximum-likelihood estimates (MLE) of the parameters of the model. The details of the MLE method applied to this response model are explained in Rypdal and Rypdal (2013). In a short-range memory response model, the powerlaw kernel $(t-s)^{\beta / 2-1}$ in the response model is replaced with an exponential $e^{-(t-s) / \tau}$, where $\tau$ is the time constant. In this case the parameter $\mu^{-1}$ can be interpreted as the effective heat capacity of the climate system. In the LRM response model $\mu^{-1}$ does not have a simple physical interpretation, although it is (in combination with $\beta$ ) a measure of the thermal inertia of the system. The memory parameter $\beta$ estimated from this model should be interpreted as the LRM parameter for the internal temperature response, and hence the problem of separating the LRM contribution from the forcing and the internal LRM has been eliminated. The $\beta$ estimated in Rypdal and Rypdal (2013) is $\beta \approx 0.75$, which is not much lower than the value estimated for the full temperature record from detrending techniques like DFA and WVA. This shows that the detrending techniques effectively eliminate the contribution to $\beta$ from the anthropogenic trend.

For the response model analysis in the present paper, all forced temperature time series are provided together with time series for the solar, volcanic and greenhouse gas forcings. Other forcings may be included in the model simulations, but they are not included in the analysis. If the exact forcings used in the model experiments are not available, the forcing described in Crowley (2000) is used, hereafter referred to as the "Crowley forcing". For the ECHO$\mathrm{G}$ forced run and the Moberg reconstructed temperature (Moberg et al., 2005), the Crowley forcing is applied. For the forced runs by COSMOS and HadCM3, the prescribed model forcings were used as input to our response model. For the LOVECLIM experiment, solar and volcanic forcings prescribed for the model were used together with $\mathrm{CO}_{2}$ and tropospheric aerosol forcings corresponding to the Crowley forcing. The full forcing data in these four cases are shown in Fig. 1.
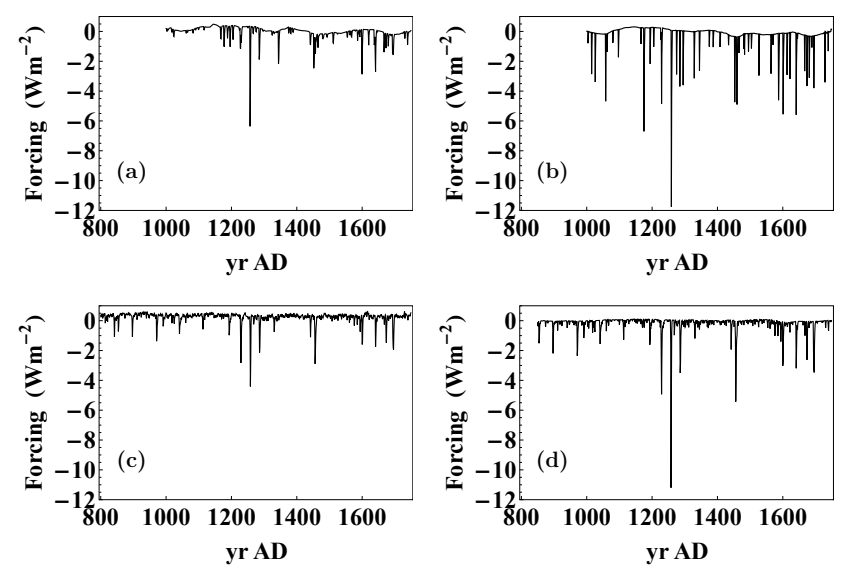

Figure 1. The different forcings used as input to the response model: (a) Crowley forcing used with the Moberg reconstruction and the ECHO-G simulation, (b) forcing used in the COSMOS experiment, (c) in the LOVECLIM experiment and (d) in the HadCM3 experiment.

\section{Data}

\subsection{The reconstruction of Moberg et al. (2005)}

The reconstructed temperature presented in Moberg et al. (2005) is a $\mathrm{NH}$ reconstruction covering the time period AD 1-1979. The reconstruction is created from 11 lowresolution proxy time series (e.g. ice cores and sediments, 1-180 year resolution) and seven tree-ring records (annual resolution). The 18 local reconstructed temperature time series were first divided into an Eastern and a Western part. Linear interpolation was then applied to all time series in order to create annual mean values. The beginning and end of the time series were padded with surrogate data so that they all covered the time period $300 \mathrm{BC}-\mathrm{AD} 2300$ to minimize edge effects of the wavelet transform. The wavelet transform (WT) with the Mexican hat wavelet basis function was then applied using the set of 22 scales to generate 22 time series. For each scale 1-9 (Fourier timescales $<80$ years), the WTs from the tree-ring proxy series were averaged. For the scales 10-22 (Fourier timescales $>80$ years), the WTs from the low-resolution proxy series were averaged. Scale 1-22 were then merged, creating two full WT time series, one for the Eastern and one for the Western NH. The two subsets were then averaged, and the inverse WT was calculated, creating a dimensionless $\mathrm{NH}$ temperature reconstruction. Finally, the mean and variance of the reconstructed temperature time series were calibrated to correspond to the instrumental data available for the time period 1856-1978.

\subsection{Marine sediment SST reconstruction; Reykjanes Ridge}

The local sea surface temperature (SST) reconstruction applied in the following study is presented in detail in Miettinen 
et al. (2012). Past August SST has been reconstructed by analysing marine planktonic diatoms from a composite marine sediment core, recovered at the Reykjanes Ridge in the western subpolar North Atlantic $\left(57^{\circ} 27.09^{\prime} \mathrm{N}, 27^{\circ} 54.53^{\prime} \mathrm{W}\right.$, at $2630 \mathrm{~m}$ water depth). The composite core consists of a $54.3 \mathrm{~cm}$ long box core, and a $3.725 \mathrm{~m}$ long gravity core. The general assumption is that the down-core diatomic microfossil assemblages are related with past environmental conditions at the core site. Marine diatoms are unicellular, photosynthetic algae with siliceous frustules. For this particular analysis, the down-core diatomic assemblages were converted to August SST estimates by the weighted-average partial least squares technique of ter Braak and Juggins (1993). The SST reconstruction has an average temporal resolution of 2 years for year 1770-2000 (box core), and 8-10 years for year 1000-1770 (gravity core).

\subsection{SST reconstruction from observations; Reykjanes Ridge}

A reconstruction based on instrumental observations was developed in Smith and Reynolds (2005). For the ocean, SST was used, while surface marine air temperatures where left out due to biases in the daytime temperatures. The SST analysis and a separate land surface air temperature analysis were merged to form a monthly merged analysis from 1880 to 1997. The International Comprehensive Ocean-Atmosphere Data Set (ICOADS) SST observations release 2 was the primary SST data, but the combined satellite and in situ SST analysis of Reynolds et al. (2002) was also included. The reconstruction was separated into low- and high-frequency components, which were added for the total reconstruction. The low frequency was reconstructed using spatial and temporal filtering, with a time filter of 15 years. The lowfrequency component was subtracted from the data before reconstruction of the high-frequency component using spatial covariance modes. The method for reconstructing the data is described in detail in Smith and Reynolds (2004). This reconstruction contains improvements over many earlier studies: It is globally complete, incorporates updates in ICOADS, the analysis variance have less dependence on sampling compared to some earlier analysis, and uncertainty estimates indicate when and where the analysis is most reliable.

\subsection{LOVECLIM model and experiment}

The Earth system model LOVECLIM version 1.2 contains a quasi-geostrophic model for the atmosphere (ECBilt2), coupled to an ocean GCM (CLIO3) (Goosse et al., 2010). The two models have 3 and 20 vertical levels, respectively. A thermodynamic sea-ice model is incorporated into the OGCM, and the vegetation model VECODE is used to simulate the dynamics of trees, grasses and deserts. It includes the evolution of the terrestrial carbon cycle, while a separate model LOCH simulates the ocean carbon cycle. Both the solubility and the biological pumps are included in this model. Incorporated in LOVECLIM is also the ice-sheet model AGISM, which consists of three modules: ice sheet flow, visco-elastic bedrock and mass balance at the ice-atmosphere and iceocean interfaces.

We apply surface temperature data from one experiment with this model; "LOVECLIM Climate Model Simulation Constrained by Mann et al. (2009) Reconstruction" (Goosse et al., 2012). In this experiment, simulations are constrained by the mean surface temperature reconstruction of Mann et al. (2009). External forcing includes TSI (total solar irradiance), volcanic eruptions, land cover changes, orbital forcing, greenhouse gases and aerosols. When we implement the response model to these data, time series for the solar, volcanic and greenhouse gas forcing are applied. The solar forcing time series is based on the reconstruction by Muscheler et al. (2007). The volcanic activity time series originate from Crowley et al. (2003), while the greenhouse gas forcing used is obtained from Crowley (2000).

\subsection{COSMOS ESM model and experiments}

The COSMOS ESM model consists of GCMs for the atmosphere and the ocean (Jungclaus et al., 2010). The atmospheric model ECHAM5 (Roeckner et al., 2003) has 19 vertical levels, while the ocean model MPIOM (Marsland et al., 2003) has 40. A thermodynamic sea-ice model is incorporated into the OGCM. Additional modules include the ocean biogeochemistry model HAMOCC5 (Wetzel et al., 2006), and the terrestrial biosphere model JSBACH (Raddatz et al., 2007).

The surface temperature data applied in our analysis are extracted from one experiment in a set of experiments referred to as "Ensemble Simulation of the Last Millennium using the Comprehensive COSMOS Earth System Model" (Jungclaus et al., 2010). External forcing used in the forced simulations include TSI, volcanoes, orbital forcing, greenhouse gases and land use change. An unforced control run is also included in the comparative LRM study.

For the response model, time series for solar, volcanic and greenhouse gas forcing are applied. The forcing time series used are created specifically for this model and experiment (Jungclaus et al., 2010). The solar forcing time series is based on a combination of reconstructions; from the Maunder Minimum (AD 1647-1715) until today the total solar irradiance (TSI) is based on historical sunspot records (Krivova and Solanki, 2007; Balmaceda et al., 2007), and between 800 AD and the Maunder Minimum the TSI is reconstructed from estimates of the solar open magnetic flux based on ${ }^{14} \mathrm{C}$ concentrations in tree rings (Solanki et al., 2004; Krivova and Solanki, 2008; Usoskin et al., 2011). An 11-year solar cycle has been superposed on this part of the reconstruction.

The relative radiative forcing from volcanic eruptions is calculated from aerosol optical depth (AOD) and effective radius $R_{\text {eff }}$. Satellite data from the 1991 Mt Pinatubo eruption 
is the basis for these estimates. The greenhouse gas forcing includes $\mathrm{CO}_{2}$, where concentrations are computed within the model, based on historical records of fossil fuel emissions by Marland et al. (2003).

\subsection{ECHO-G model and experiments}

The coupled model ECHO-G (Legutke and Voss, 1999) version 4 consists of GCMs for the ocean/sea ice and the atmosphere. The atmospheric model ECHAM4 (Roeckner et al., 1996) includes 19 vertical levels, while the ocean model HOPE-G (Legutke and Maier-Reime, 1999) includes 20 levels. External forcing includes volcanoes, solar irradiance and greenhouse gases. Suitable time series for the model forcings were not available, and for this reason the Crowley (2000) forcings are used in the response model study. Surface temperature from two experiments is used for analysis: one forced run and one control run with forcing values fixed to year 1990 (Zorita et al., 2003; González-Rouco et al., 2003; von Storch et al., 2004).

\subsection{HadCM3 model and experiment}

The Hadley Centre coupled model 3 is an AOGCM (Gordon et al., 2000), with 19 levels in the atmospheric component HADAM3 (Pope et al., 2000) and 20 levels on the ocean HADOM3 component. External forcing includes volcanoes, solar irradiance, greenhouse gases, land use change and orbital variations. In the response model study, the time series for volcanic forcing (Crowley et al., 2008), solar irradiance (Steinhilber et al., 2009) and greenhouse gases (Schmidt et al., 2011) from the model experiment were applied. The complexity, time period covered, and temporal and spatial resolution for each model experiment are given in Table 1.

\section{Results}

When applying periodogram, DFA2 and WVA2 directly to the temperature reconstruction records, all data up to the year 1750 are used. Because the Crowley forcing record starts at AD 1000, only data from this year and forward were used in the response model residual analysis of the Moberg reconstructed temperature and the temperature from the LOVECLIM experiment. Therefore the scales shown in the plots may differ somewhat between the full record and the residual from the deterministic response. Table 2 shows the resulting $\beta$ from applying periodogram, DFA2 and WVA2 directly to the full temperature and to the residuals from the deterministic response. The $\beta$ estimated using the response model is also given, where the parameters of the response model are estimated with the MLE method (Rypdal and Rypdal, 2013). The response model residual analysis is applied to the temperature reconstructions and the temperature from forced climate model experiments, while the direct analysis also includes control simulations. The scaling ranges used to find $\beta$
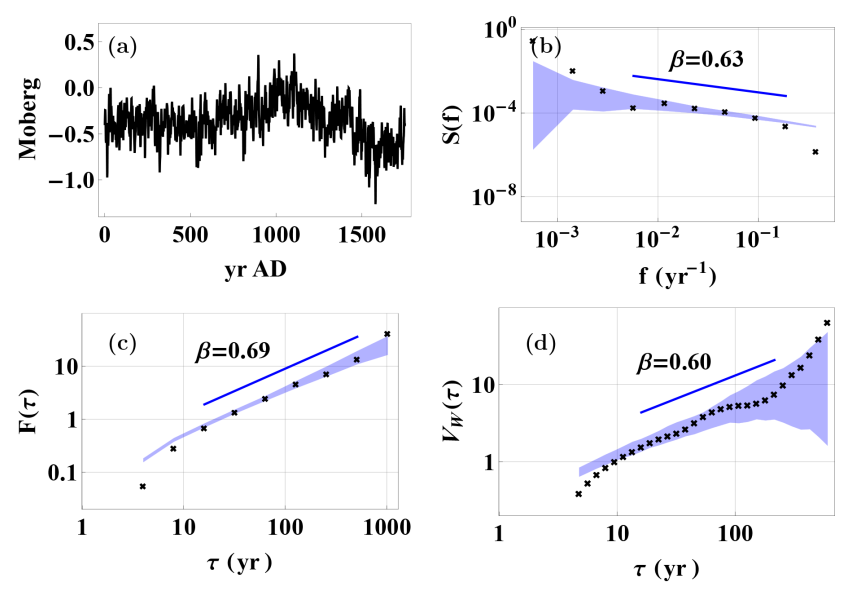

Figure 2. (a) The Moberg reconstructed temperature. (b) PSD, (c) DFA2, and (d) WVA2 applied to the record. The shaded areas are the $95 \%$ confidence range for synthetic fGn with $\beta$ estimated by PSD, DFA2 and WVA2. The $\beta$-values are estimated from the slopes of the straight lines and the range of scales used for estimation is indicated by the length and position of these line segments. The estimated $\beta$-values are indicated in the figure.

are shown in the figures and not given in the table. Figures 213 show the analysis of the temperature records. For the full records, the figures show (a) the temperature data, and (b) PSD, (c) DFA2, and (d) WVA2 applied to the data set. For the response model results, the figures show (a) the temperature data and deterministic response, (b) the residual, and (c) PSD and (d) DFA2 applied to the residual. The residual is the deterministic response interpolated to have the original time resolution subtracted from the full-resolution temperature data; $95 \%$ confidence areas are shown for all three methods, computed from Monte Carlo ensembles of synthetic fGns. For the response-model analysis with PSD and DFA2, the synthetic fGns are generated with the $\beta$-value estimated by MLE, otherwise they are generated with $\beta$ found from fitting straight lines to the log-log plots of the PSD, DFA2 and WVA2 analysis, respectively. The values of $\beta$ used are indicated in each figure.

We have not given error bars on the estimated $\beta$-values in each case. Such error bars can easily be estimated from fitting straight lines to each realization in the Monte Carlo ensembles, and computing probability density functions (PDFs) of their slopes. This was done by Rypdal et al. (2013) for time series samples of similar lengths as studied here and error bars of $\Delta \beta= \pm 0.2$ (95\% confidence) was generally found for all estimation methods. Hence, even though the $\beta$ estimates in this paper are given with two decimals, the last decimal is hardly significant. The discrepancies in the $\beta$ estimates between the methods are within these error bars, and hence there is no point in trying to explain these differences. 
Table 1. Information on temperature from model experiments

\begin{tabular}{lllll}
\hline Climate model & LOVECLIM ESM v. 1.2 & COSMOS ESM & ECHO-G & HadCM3 \\
\hline Complexity & interm. & GCM & GCM & GCM \\
Time period covered & AD 500-1750 & AD 800-1750 & AD 1000-1750 & AD 850-1750 \\
Temp. res. & annual & monthly & monthly & monthly \\
Spat. res. & $5.63 \times 5.63$ degrees & $3.75 \times 3.75$ degrees & $3.75 \times 3.75$ degrees & $2.55 \times 3.75$ degrees
\end{tabular}

Table 2. Estimated $\beta$ from applying the PSD, DFA 2 and WVA2 directly to the full temperature record (all temperatures), from PSD, DFA2 and WVA2 applied to the residuals from the deterministic response, and from the response model using MLE (temperature reconstruction and temperature from the forced climate model run experiments). The error bars on all estimates are approximately $\Delta \beta \pm 0.2$ (see main text).

\begin{tabular}{lccccccc}
\hline & \multicolumn{3}{c}{ Full record } & \multicolumn{3}{c}{ Residual } & \multicolumn{2}{c}{ Response model } \\
& PSD & DFA2 & WVA2 & PSD & DFA2 & WVA2 & MLE \\
\hline Moberg & 0.63 & 0.69 & 0.60 & 0.75 & 0.59 & 0.42 & 0.75 \\
LOVECLIM forced & 1.10 & 0.98 & 0.96 & 1.07 & 1.01 & 0.97 & 0.95 \\
COSMOS forced & 0.90 & 0.82 & 0.79 & 0.88 & 0.77 & 0.72 & 0.61 \\
COSMOS ctrl & 0.87 & 0.82 & 0.86 & & & & \\
ECHO-G forced & 0.99 & 0.91 & 0.90 & 0.86 & 0.72 & 0.84 & 0.75 \\
ECHO-G ctrl & 0.85 & 0.85 & 0.87 & & & & \\
HadCM3 forced & 1.14 & 1.12 & 1.17 & 0.89 & 0.90 & 0.86 & 0.82 \\
\hline
\end{tabular}
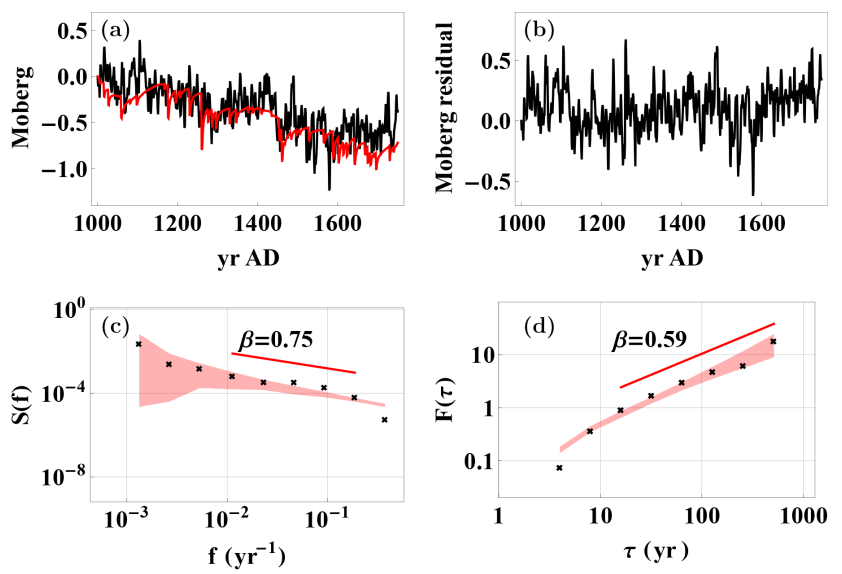

Figure 3. (a) The Moberg reconstructed temperature (black) and the deterministic response (red). (b) The residual from the deterministic response. (c) PSD from the residual (black crosses) and linear fit to the PSD in the scale range indicated by the red line segment. The value $\beta=0.75$ estimated from this line segment is indicated in the figure. (d) DFA2 applied to the residual (black crosses). The straight line segment is a linear fit to the dots in the range indicated by the length, and position of the line segment and the slope yields an alternative estimate $\beta=0.59$ for the residual, indicated in the figure. The shaded area in (c) and (d) is the $95 \%$ confidence range for synthetic fGn with $\beta=0.75$ estimated by MLE using the response model.
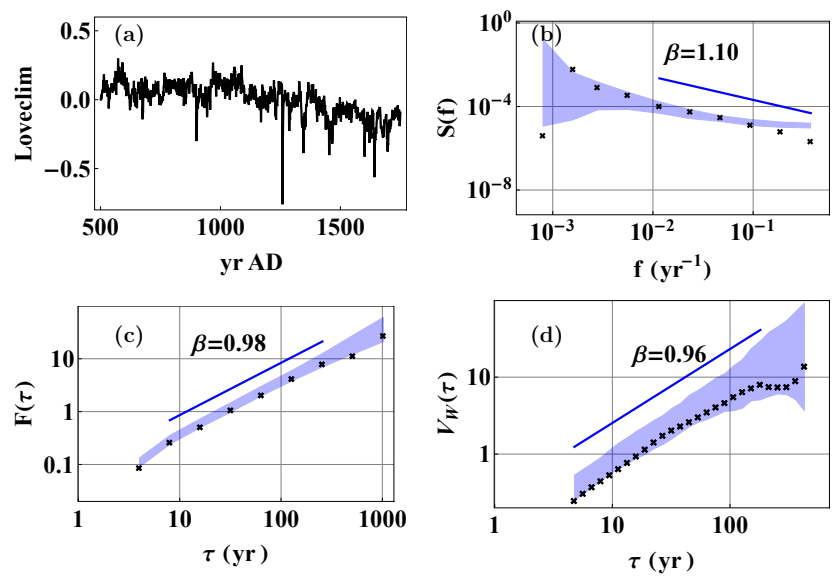

Figure 4. (a) The temperature from the LOVECLIM experiment. (b) PSD, (c) DFA2, and (d) WVA2 applied to the record. The shaded areas are the $95 \%$ confidence range for synthetic fGn with $\beta$ estimated by PSD, DFA 2 and WVA2. The $\beta$-values are estimated from the slopes of the straight lines and the range of scales used for estimation is indicated by the length and position of these line segments. The estimated $\beta$-values are indicated in the figure.

\subsection{Results from palaeoreconstruction of Moberg et al. (2005)}

Figure 2 shows the Moberg reconstructed temperature record, its power spectral density (PSD) and the results of DFA2 and WVA2 applied to the full record. As discussed above, the scales up to $\sim 10$ years are not representative for the temperature, and this is seen as a cross-over in the slope 

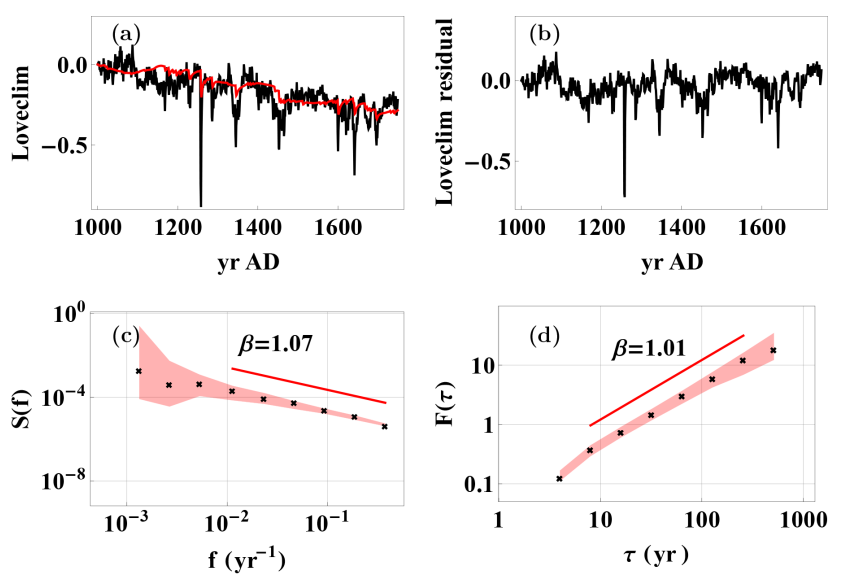

Figure 5. (a) Temperature from the LOVECLIM experiment with 1-year resolution (black) and deterministic response (red). (b) The residual from the deterministic response. (c) PSD from the residual (black crosses) and linear fit to the PSD in the scale range indicated by the red line segment. The value $\beta=1.07$ estimated from this line segment is indicated in the figure. (d) DFA2 applied to the residual (black crosses). The straight line segment is a linear fit to the dots in the range indicated by the length, and position of the line segment and the slope yields an alternative estimate $\beta=1.01$ for the residual, indicated in the figure. The shaded area in (c) and (d) is the $95 \%$ confidence range for synthetic fGn with $\beta=0.95$ estimated by MLE using the response model.

of the log-log periodogram and DFA2 and WVA2 fluctuation functions. The deviation from a straight line at the largest scales (lowest frequencies), which is most prominent in the WVA2 fluctuation function, is caused by a nonlinear trend associated with the two well-known climatic features of the last 2000 years: the Medieval climate anomaly and the Little ice age. The three methods yield $\beta \approx 0.63, \beta \approx 0.69$ and $\beta \approx 0.60$, respectively.

In principle the LRM properties due to internal dynamics can be separated from those induced by the external forcing by applying the response model method of Rypdal and Rypdal (2013) described in Sect. 2. This method allows estimation of the model parameters $\beta, \sigma$ and $\mu$ from the Crowley forcing data and the Moberg reconstruction record. Then we can compute the deterministic response and the residual obtained by subtracting this deterministic response from the Moberg record. The residual represents the response to the stochastic forcing and hence the internal variability of the climate system. The scaling properties of this residual can be assessed with the periodogram or the DFA method which also provides a consistency test on the maximum-likelihood estimate of $\beta$.

A caveat here is the low-pass filtered nature of the Moberg record. The MLE method tends to emphasize the shorter scales on which the reconstruction record is smooth, and this will spuriously yield $\beta \approx 1$. A way to avoid this could be to coarse grain both temperature and forcing time series by av-
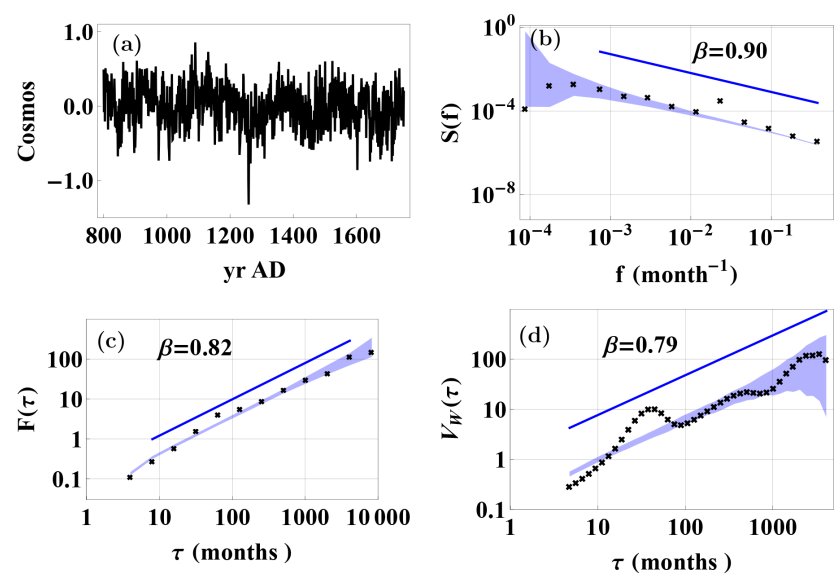

Figure 6. (a) The temperature from the COSMOS experiment. (b) PSD, (c) DFA2, and (d) WVA2 applied to the record. The shaded areas are the $95 \%$ confidence range for synthetic fGn with $\beta$ estimated by PSD, DFA 2 and WVA2. The $\beta$-values are estimated from the slopes of the straight lines and the range of scales used for estimation is indicated by the length and position of these line segments. The estimated $\beta$-values are indicated in the figure.

eraging over successive time windows of a certain length $t_{A}$, such that the temperature series is no longer smooth. This will give a more reasonable maximum-likelihood estimate of $\beta$, but the coarse-grained data cannot capture the causal connection between the almost instantaneous volcanic forcing spikes and the temperature response to them. The resulting blurring of the causal connection on timescales shorter than a decade has the effect that the MLE method interprets the variability on these short scales as stochastic, and hence overestimates the stochastic forcing strength $\sigma$, and also yields incorrect estimates of $\mu$ and $\beta$. The lesson to learn from this is that we cannot expect to obtain a correct separation of deterministic and stochastic forcing and correct parameter estimates from the low-resolution reconstruction data. Another approach to circumvent this problem was suggested in Rypdal and Rypdal (2013), where the response model parameters computed from the annual-resolution instrumental data were applied to the millennium-long annual-resolution forcing record to produce a deterministic-response record with annual resolution. The Moberg record and this deterministic response is shown in Fig. 3a. The residual obtained by subtracting the deterministic response from the reconstructed record is shown in Fig. 3b, and provides a good representation of the internal variability on timescales longer than a decade. On shorter timescales the residual is strongly influenced by the forced response due to the smooth character of the temperature reconstruction, but we do not need to use those scales to estimate model parameters if we do not insist on using MLE. The PSD of the residual is shown to exhibit good scaling in Fig. 3c, and the DFA2 fluctuation function of this residual on the longer timescales should provide good estimates of $\beta$ for the internal variability, as shown in Fig. 3d. 

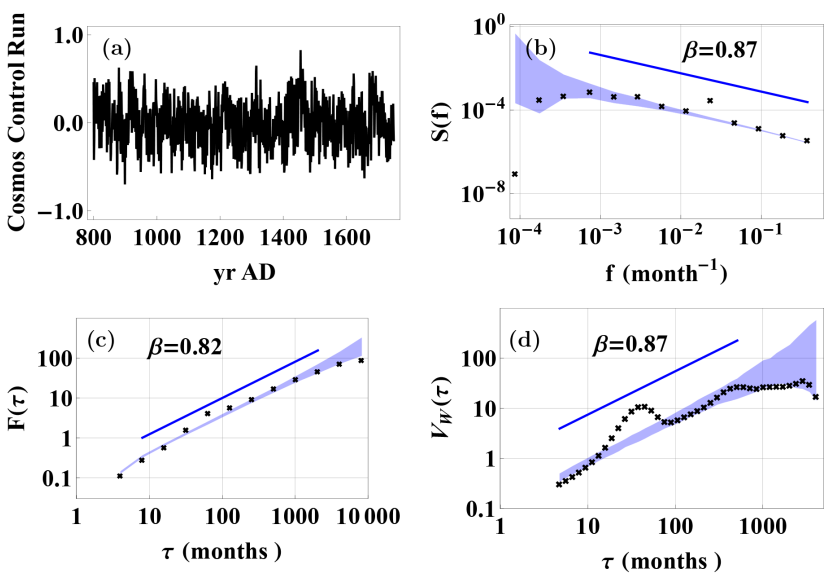

Figure 7. (a) The temperature from the COSMOS control simulation. (b) PSD, (c) DFA2, and (d) WVA2 applied to the record. The shaded areas are the $95 \%$ confidence range for synthetic fGn with $\beta$ estimated by PSD, DFA 2 and WVA2. The $\beta$-values are estimated from the slopes of the straight lines and the range of scales used for estimation is indicated by the length and position of these line segments. The estimated $\beta$-values are indicated in the figure.

\subsection{Results from LOVECLIM experiment}

The NH temperature record for the period AD 1000-1750 for the LOVECLIM experiment, its power spectral density (PSD), and the DFA2 and WVA2 fluctuation functions are shown in Fig. 4. There appears to be good scaling with $\beta \approx 1$ at least on timescales up to a few hundred years. The response model gives similar value of $\beta$, which suggests that the persistence observed in the modelled record is due to LRM in the internal dynamics and not a reflection of LRM in the forcing. In this model simulation both forcing input and simulation output are given with annual resolution. This allows us to handle volcanic forcing and the response to volcanic eruptions in a realistic manner. The results from the response model estimates with annual resolution are shown in Fig. 5.

\subsection{Results from COSMOS experiment}

The temperature from the COSMOS forced run experiments exhibits some oscillations. In particular, a prominent peak corresponding to a multiannual mode is seen in the PSD in Fig. 6b, and in the WVA plot in Fig. 6d. Otherwise scaling is fairly good with $\beta \approx 0.8-0.9$. For the control simulation (Fig. 7) $\beta$ is almost the same as in the forced simulation, but PSD, DFA2, and WVA2 show some signs of loss of memory on scales longer than a century. The multiannual oscillation influences the maximum-likelihood estimation of model parameters in the response model, so in Fig. 8 these estimates have been performed on a 4-year coarse-grained time series, while the residual has been computed on monthly scale. As discussed above, such coarse-graining creates a misrepresen-
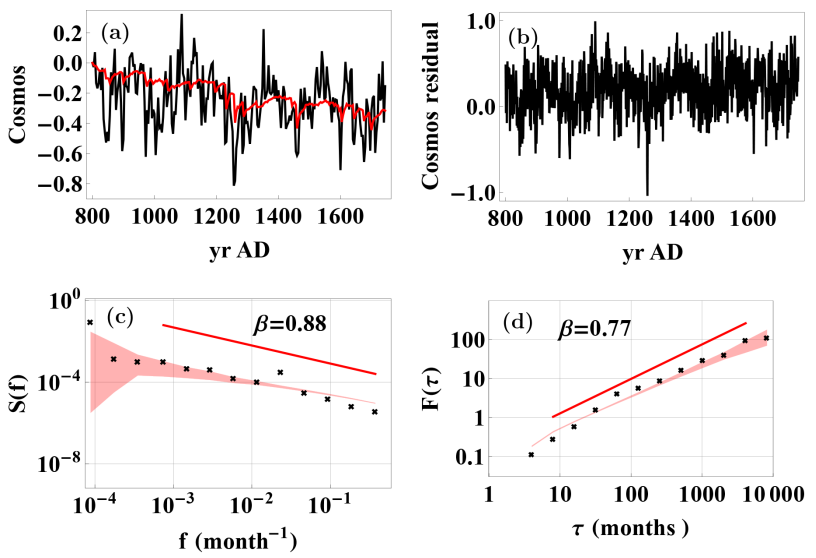

Figure 8. (a) Four-year average of the temperature from the COSMOS experiment (black) and deterministic response (red). (b) The residual from the deterministic response. (c) PSD from the residual (black crosses) and linear fit to the PSD in the scale range indicated by the red line segment. The value $\beta=0.88$ estimated from this line segment is indicated in the figure. (d) DFA2 applied to the residual (black crosses). The straight line segment is a linear fit to the dots in the range indicated by the length, and position of the line segment and the slope yields an alternative estimate $\beta=0.77$ for the residual, indicated in the figure. The shaded area in (c) and (d) is the $95 \%$ confidence range for synthetic fGn with $\beta=0.61$ estimated by MLE using the response model.
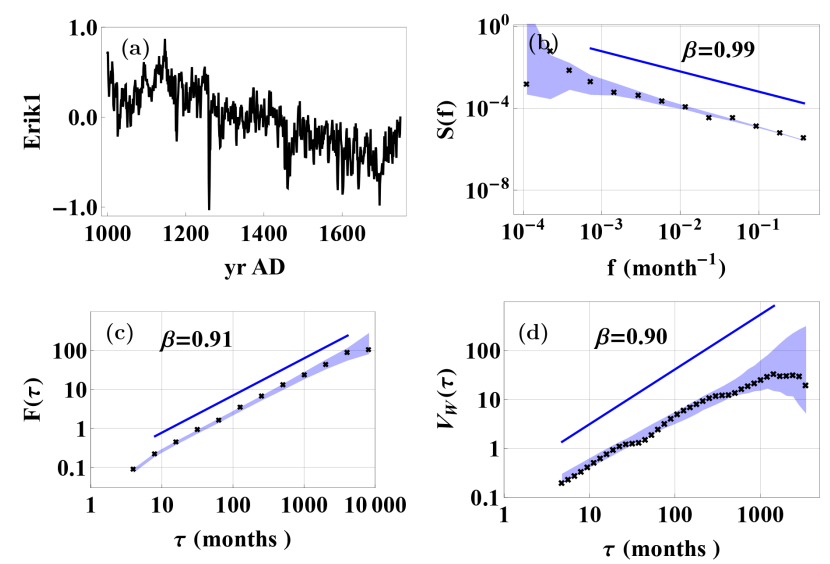

Figure 9. (a) The temperature from the ECHO-G experiment Erik1. (b) PSD, (c) DFA2, and (d) WVA2 applied to the record. The shaded areas are the $95 \%$ confidence range for synthetic fGn with $\beta$ estimated by PSD, DFA2 and WVA2. The $\beta$-values are estimated from the slopes of the straight lines and the range of scales used for estimation is indicated by the length and position of these line segments. The estimated $\beta$-values are indicated in the figure.

tation of the response to volcanic eruptions, which we believe is the main reason for the lower estimate of $\beta \approx 0.6$ from the response model.

In a recent paper Henriksson et al. (2014) analyse COSMOS global temperature series and obtain lower spectral exponents than we do, in particular for the unforced 

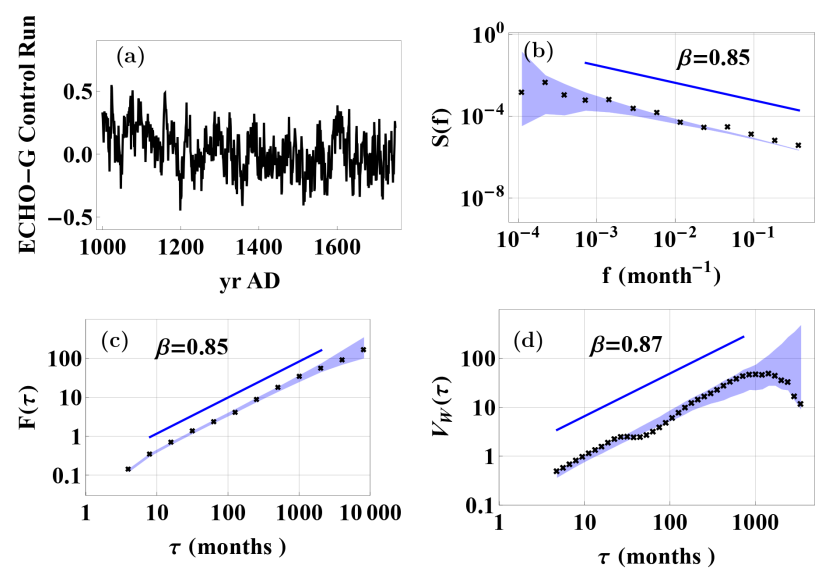

Figure 10. (a) The temperature from the ECHO-G control simulation. (b) PSD, (c) DFA2, and (d) WVA2 applied to the record. The shaded areas are the $95 \%$ confidence range for synthetic fGn with $\beta$ estimated by PSD, DFA 2 and WVA2. The $\beta$-values are estimated from the slopes of the straight lines and the range of scales used for estimation is indicated by the length and position of these line segments. The estimated $\beta$-values are indicated in the figure.

simulation. The reasons for this discrepancy are the following: the COSMOS simulation has a very strong El Niño-Southern Oscillation (ENSO)-like signal, considerably stronger than in instrumental or reconstruction data. This leads to a distinct spectral "hump" in the global temperature on frequencies in the range $1 / 6<f<1$ year $^{-1}$, but with a long tail towards lower frequencies. The spectral shape of the ENSO signal is apparent from their spectra from locations in the tropical oceans while away from this region there is a much wider power-law range with $\beta \approx 1$. The globally averaged temperature is a superposition of signals having these two spectral shapes, and the result is that the range of frequencies $f<1 / 6$ year $^{-1}$ are influenced both by the ENSO hump and the underlying power law which dominates at higher latitudes. This leads to an apparent lower slope (lower $\beta$ ) of the log-log spectrum in this range. Henriksson et al. (2014) make a point of splitting the spectrum of a non-scaling global signal into short pieces (ranges) and compute slopes for each of these ranges. We cannot see that this has physical meaning, since what appears as different ranges are results of combining (averaging over) signals with different scaling properties - in this case power-law scaling signals from temperate and polar zones, and quasi-oscillatory signals from the tropical oceans.

The goal of our analysis has been to uncover the underlying power-law scaling which prevails away from the ENSO region, and is why we have focused on DFA, which is relatively insensitive to oscillatory perturbations. In comparison, the WVA is much more sensitive to the ENSO perturbation. However, the underlying scaling also becomes apparent from the power spectrum if we analyse monthly, deseasonalized, data, as shown in Figs. 6b, $7 \mathrm{~b}$ and 8c.
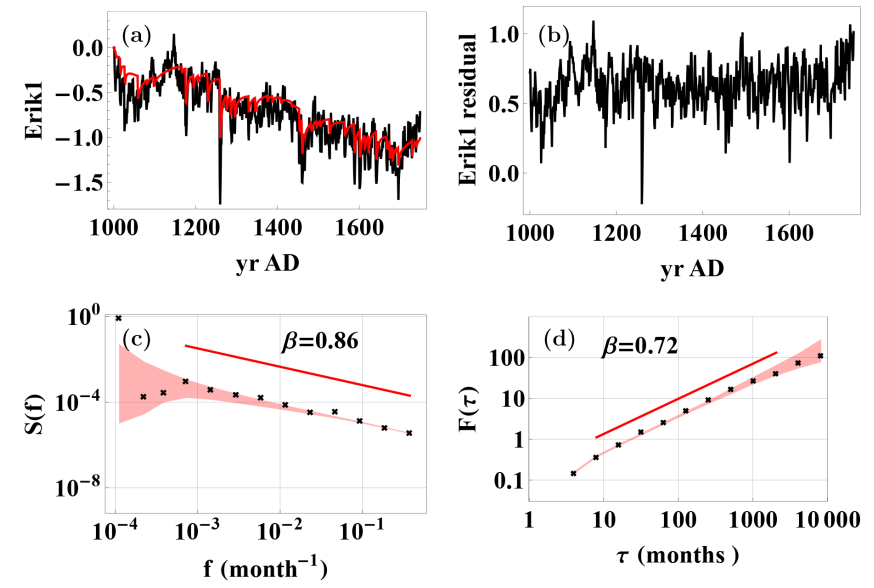

Figure 11. (a) One-year average of the temperature from the experiment Erik1 (black) and deterministic response (red). (b) The residual from the deterministic response. (c) PSD from the residual (black crosses) and linear fit to the PSD in the scale range indicated by the red line segment. The value $\beta=0.86$ estimated from this line segment is indicated in the figure. (d) DFA2 applied to the residual (black crosses).The straight line segment is a linear fit to the dots in the range indicated by the length, and position of the line segment and the slope yields an alternative estimate $\beta=0.72$ for the residual, indicated in the figure. The shaded areas in (c) and (d) show the $95 \%$ confidence range for synthetic fGn with $\beta=0.74$ estimated by MLE using the response model.
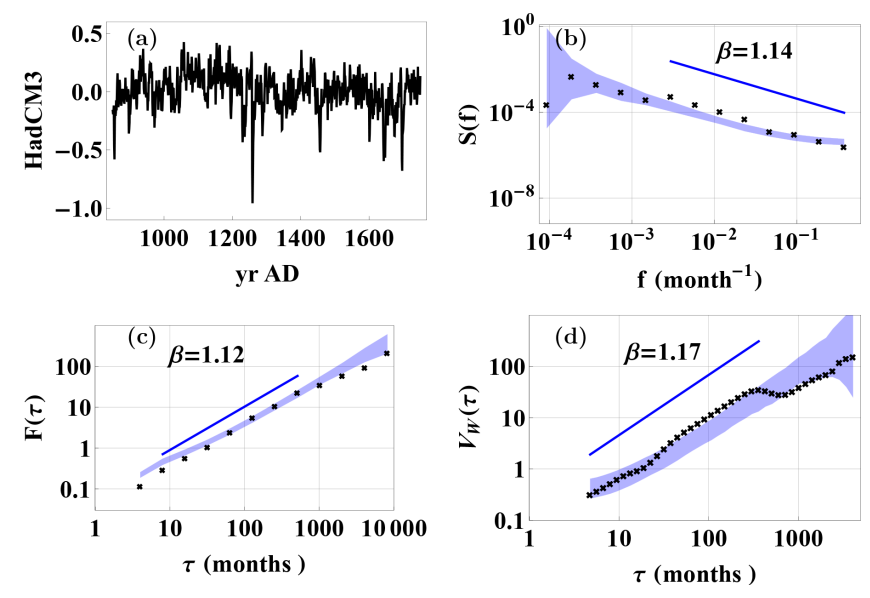

Figure 12. (a) The temperature from the HadCM3 experiment. (b) PSD, (c) DFA2, and (d) WVA2 applied to the record. The shaded areas show the $95 \%$ confidence range for synthetic fGn with $\beta$ estimated by PSD, DFA2 and WVA2. The $\beta$-values are estimated from the slopes of the straight lines and the range of scales used for estimation is indicated by the length and position of these line segments. The estimated $\beta$-values are indicated in the figure.

\subsection{Results from ECHO-G experiments}

The temperature from the forced experiment "Erik1" shows good scaling with $\beta \approx 0.9$ in Fig. 9. The temperature from the control run (Fig. 10) also scales well with a similar 

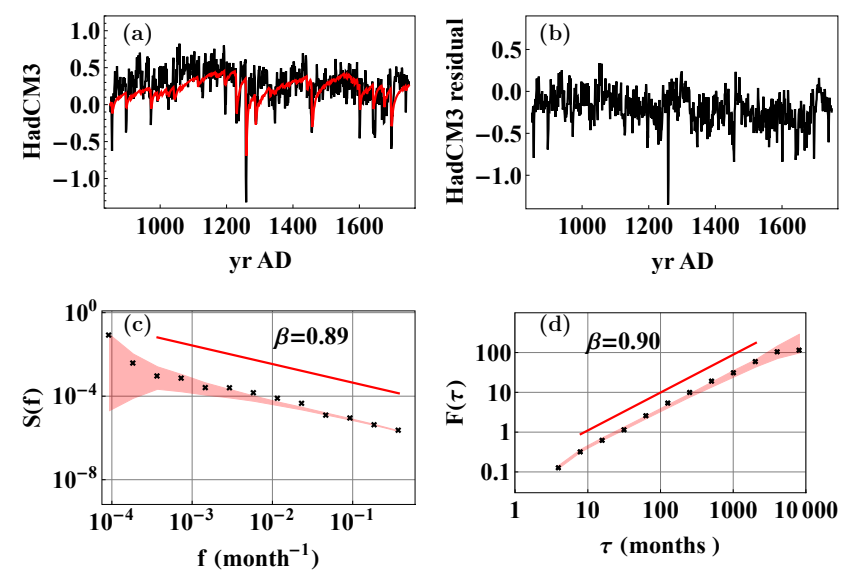

Figure 13. (a) The temperature from the HadCM3 experiment (black) and deterministic response (red). (b) The residual from the deterministic response. (c) PSD from the residual (black crosses) and linear fit to the PSD in the scale range indicated by the red line segment. The value $\beta=0.89$ estimated from this line segment is indicated in the figure. (d) DFA2 applied to the residual (black crosses). The straight line segment is a linear fit to the dots in the range indicated by the length, and position of the line segment and the slope yields an alternative estimate $\beta=0.90$ for the residual, indicated in the figure. The shaded areas in (c) and (d) show the $95 \%$ confidence range for synthetic fGn with $\beta=0.82$ estimated by MLE using the response model.

value for $\beta$. The response model yields a slightly smaller $\beta$ (Fig. 11). Here a 1-year coarse graining has been applied before the parameters have been estimated, since the forcing data have 1-year resolution.

\subsection{Results from the HadCM3 experiment}

The HadCM3 experiment consists of only a forced simulation, and the scaling properties of the $\mathrm{NH}$ temperature series from this experiment differ from the other experiments in some respects. Figure 12 shows a $\beta$ slightly greater than 1 but a cross-over to a regime with lower $\beta$ regimes for $\tau \sim 30$ year. The analysis with the response model, however, indicates a more consistent scaling with $\beta \approx 0.8-0.9$, as shown in Fig. 13. The discrepancy between the scaling of the forced signal and the residual is attributed to the strong solar forcing signal employed in the HadCM3 simulation.

\subsection{Scaling in local data; Reykjanes Ridge}

In our experience, analysis of instrumental local station data from continental interiors typically yields very low persistence on timescales up to a few decades. On the other hand, coastal and oceanic observations in the temperate regions of the NH present persistent $\beta$-values closer to those found for the hemispheric average. We also believe that this feature extends beyond the decadal scales, i.e. that good scaling with strong persistence prevails on scales up to several centuries in

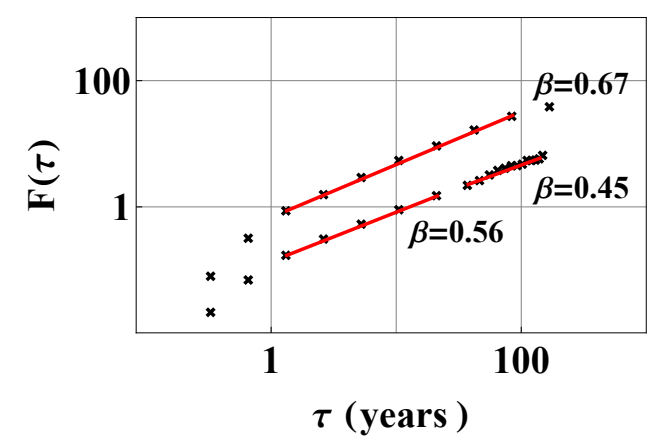

Figure 14. DFA2 applied to sea surface or air surface temperature at Reykjanes Ridge. The upper curve is the result for the air surface temperature from Erik1 experiment. The lower left curve is based on the monthly reconstructed data for sea surface temperature, and the lower right curve on the marine sediment reconstruction of ocean temperature. The red lines indicate the scales used to estimate $\beta$.

the northern oceans. As an illustration we present in Fig. 14 analysis of the Reykjanes Ridge reconstruction from marine sediments described in Section 3.2 and the ECHO-G Erik1 experiment for the period AD 1000-1750, and of the monthly SST reconstruction for the period 1880-1997 as described in Sect. 3.3. The figure shows DFA2 plots for the three data sets. ECHO-G shows good scaling in the range 1-100 years with $\beta \approx 0.67$, as compared to $\beta=0.91$ for the $\mathrm{NH}$ average. The marine sediment reconstruction yields $\beta \approx 0.45$, and the instrumentally based reconstruction $\beta \approx 0.56$. The greatest uncertainty in the $\beta$-estimate is in the marine sediment reconstruction, for which a very limited range of scales is available for analysis. The record has uneven time spacing, but the time step is mostly almost the same, slightly below 10 years. The data points inconsistent with this are ignored, and DFA2 applied to the remaining record. A maximum-likelihood estimation method for time series with uneven time spacing yields $\beta$ close to what was found with DFA2. In spite of these uncertainties the analysis demonstrates consistently persistent scaling over timescales from years to centuries in these local data from model experiment and reconstruction data.

\section{Conclusions}

The temperatures from all model experiments yield higher values of $\beta$ than the Moberg reconstruction when scales longer than a decade are considered. The reconstruction is stated to represent temperature in the $\mathrm{NH}$, but most of the proxies used are in land and coastal areas. In this sense they may be considered more like representations of land or coastal temperature. Studies of observational data show higher persistence in SST than land air temperature, and the value for $\beta$ found for the Moberg record is more in agreement with the one found for the NH land temperature than ocean temperature (Eichner et al., 2003; Lennartz and Bunde, 2009a). Our estimate is in agreement with Rybski et al. 
(2006). The temperature from the model experiments is averaged over grid cells from both land and ocean areas, and the influence of the ocean might be what yields the higher value of $\beta$ than found for the Moberg reconstruction.

The temperatures from the COSMOS experiments (both forced and control run) clearly show an influence of a quasiperiodic variability with a 2-3 year period, which can be associated with ENSO. The ECHO-G and HadCM3 experiments show less influence of this oscillation, and in LOVECLIM it is not noticeable. For the reconstructed temperature the ENSO timescales are not resolved.

The temperatures from the forced ECHO-G experiment and the LOVECLIM experiment show a more distinct Little Ice Age anomaly, in agreement with the temperature reconstruction, than the temperature from the COSMOS and HadCM3 forced-run experiment. This anomaly may also influence the estimation of $\beta$.

All the NH-averaged temperatures from forced experiments show clear persistent scaling with $0.8<\beta<1.2$ on most of the available scales, i.e. from a decade to several centuries. The control runs and the response model estimates from the forced runs, which reveal the memory properties of the internal climate dynamics, do not show systematically less persistence than obtained directly from the simulated forced temperature records (there is a difference in HadCM3, but the difference is within the error bars). This observation does not support the notion that the observed long-range memory to great extent is generated by the forcing. Such a suggestion was made by Rybski et al. (2008), based on a global map for the parameter $\alpha=(\beta+1) / 2$ computed from both forced runs and control runs of the ECHO-G model. We believe that this discrepancy is caused by the reduction of spatiotemporal noise implied in performing a $\mathrm{NH}$ average. The differences in estimated $\alpha$ between forced and unforced simulations for local data may not be in the persistence of the underlying global signal, but rather in differences related to the amplitudes of spatiotemporal modes for the two types of simulations.

Acknowledgements. For maximium-likelihood parameter estimation in the response model we have employed an R-routine developed by Ola Løvsletten. We also thank Eduardo Zorita at the HZG for providing the ECHO-G model simulations.

Edited by: H. A. Dijkstra

\section{References}

Balmaceda, L., Krivova, N. A., and Solanki, S. K.: Reconstruction of solar irradiance using the group sunspot number, Adv. Space. Res., 40, 986-989, 2007.

Blender, R. and Fraedrich, K.: Long time memory in global warming simulations, Geophys. Res. Lett., 30, 1769, doi:10.1029/2003GL017666, 2003.
Bunde, A. and Havlin, S.: Power-law persistence in the atmosphere and in the oceans, Physica A, 314, 15-24, 2002.

Bunde, A., Havlin, S., Koscielny-Bunde, E., and Schellnhuber, H.J.: Long term persistence in the atmosphere: global laws and tests of climate models, Physica A, 302, 255-267, 2001.

Crowley, T. J.: Causes of climate change over the past 1000 years, Science, 289, 270-277, 2000.

Crowley, T. J., Baum, S. K., Kim, K.-Y., Hegerl, G. C., and Hyde, W. T.: Modeling ocean heat content changes during the last millennium, Geophys. Res. Lett., 30, 1932, doi:10.1029/2003GL017801, 2003.

Crowley, T. J., G., Z., Vinther, B., Udisti, R., Kreutzs, K., Cole-Dai, J., and Castellano, E.: Volcanism and the Little Ice Age, 16, 2223, 2008.

Eichner, J. F., Koscielny-Bunde, E., Bunde, A., Havlin, S., and Schellnhuber, H.-J.: Power-law persistence and trends in the atmosphere: A detailed study of long temperature records, Phys. Rev. E, 68, 046133, doi:10.1103/PhysRevE.68.046133, 2003.

Flandrin, P.: Wavelet analysis and synthesis of fractional Brownian motion, Information Theory, IEEE Trans., 38, 910-917, 1992.

Fraedrich, K. and Blender, R.: Scaling of Atmosphere and Ocean Temperature Correlations in Observations and Climate Models, Phys. Rev. Lett., 90, 108501, doi:10.1103/PhysRevLett.90.108501, 2003.

González-Rouco, F., von Storch, H., and Zorita, E.: Deep soil temperature as proxy for surface air-temperature in a coupled model simulation of the last thousand years, Geophys. Res. Lett., 30, 2166, doi:10.1029/2003GL018264, 2003.

Goosse, H., Brovkin, V., Fichefet, T., Haarsma, R., Huybrechts, P., Jongma, J., Mouchet, A., Selten, F., Barriat, P.-Y., Campin, J.M., Deleersnijder, E., Driesschaert, E., Goelzer, H., Janssens, I., Loutre, M.-F., Morales Maqueda, M. A., Opsteegh, T., Mathieu, P.-P., Munhoven, G., Pettersson, E. J., Renssen, H., Roche, D. M., Schaeffer, M., Tartinville, B., Timmermann, A., and Weber, S. L.: Description of the Earth system model of intermediate complexity LOVECLIM version 1.2, Geosci. Model Dev., 3, 603-633, doi:10.5194/gmd-3-603-2010, 2010.

Goosse, H., Crespin, E., Dubinkina, S., Loutre, M.-F., Mann, M. E., Renssen, H., Sallaz-Damaz, Y., and Shindell, D.: The role of forcing and internal dynamics in explaining the Medieval Climate Anomaly, Clim. Dynam., 39, 2847-2866, 2012.

Gordon, C., Cooper, C., Senior, C. A., Banks, H., Gregory, J. M., Johns, T. C., Mitchell, J. F. B., and Wood, R. A.: The simulation of SST, sea ice extents and ocean heat transports in a version of the Hadley Centre coupled model without flux adjustments, Clim. Dynam., 16, 147-168, 2000.

Govindan, R. B., Vjushin, D., Brenner, S., Bunde, A., Havlin, S., and Schellnhuber, H.-J.: Long-range correlation and trends in global cliamte models: Comparison with real data, Physica A, 294, 239-248, 2001.

Govindan, R. B., Vjushin, D., Bunde, A., Brenner, S., Havlin, S., and Schellnhuber, H.-J.: Global climate Models Violate Scaling of the Observed Atmospheric Variability, Phys. Rev. Lett., 89, 028501, doi:10.1103/PhysRevLett.89.028501, 2002.

Henriksson, S., Raisanen, P., Silen, J., Jarvinen, H., and Laaksonen, A.: Improved power-law estimates from multiple samples provided by millennium climate simulations, Theor. Appl. Climatol., 1-11, doi:10.1007/s00704-014-1132-0, 2014. 
Hu, K., Ivanov, P. C., Chen, Z., Carpena, P., and Stanley, H. E.: Effect of trends on detrended fluctuation analysis, Phys. Rev. E., 64, 011114, doi:10.1103/PhysRevE.64.011114, 2001.

Jungclaus, J. H., Lorenz, S. J., Timmreck, C., Reick, C. H., Brovkin, V., Six, K., Segschneider, J., Giorgetta, M. A., Crowley, T. J., Pongratz, J., Krivova, N. A., Vieira, L. E., Solanki, S. K., Klocke, D., Botzet, M., Esch, M., Gayler, V., Haak, H., Raddatz, T. J., Roeckner, E., Schnur, R., Widmann, H., Claussen, M., Stevens, B., and Marotzke, J.: Climate and carbon-cycle variability over the last millennium, Clim. Past, 6, 723-737, doi:10.5194/cp-6723-2010, 2010.

Kantelhardt, J. W., Koscielny-Bunde, E., Rego, H. H., Havlin, S., and Bunde, A.: Detecting long-range correlations with detrended fluctuation analysis, Physica A, 295, 441-454, 2001.

Krivova, N. A. and Solanki, S. K.: Reconstruction of total solar irradiance since 1700 from the surface magnetic flux, Astron. Astrophys., 467, 335-346, 2007.

Krivova, N. A. and Solanki, S. K.: Models of solar irradiance variations: Current status, J. Astrophys. Astron., 29, 151-158, 2008.

Legutke, S. and Maier-Reime, E.: Climatology of the HOPE-G Global Ocean - Sea Ice General Circulation Model, DKRZ Tech. Rep., DKRZ-TR-21, 1999.

Legutke, S. and Voss, R.: The Hamburg Atmosphere-Ocean Coupled Circulation Model ECHO-G, DKRZ Tech. Rep.. DKRZTR-18, 1999.

Lennartz, S. and Bunde, A.: Trend evaluation in records with longterm memory: Application to global warming, Geophys. Res. Lett., 36, L16706, doi:10.1029/2009GL039516, 2009a.

Lennartz, S. and Bunde, A.: Eliminating finite-size effects and detecting the amount of white noise in short records with long-term memory, Phys. Rev. E, 79, 066101, doi:10.1103/PhysRevE.79.066101, 2009b.

Malamud, B. D. and Turcotte, D. L.: Self-affine time series: measures of weak and strong persistence, J. Stat. Plann. Inference, 80, 173-196, 1999.

Mann, M. E., Zhang, Z., Rutherford, S., Bradley, R. S., Hughes, M. K., Shindell, D., Ammann, C., Faluvegi, G., and Ni, F.: Global Signatures and Dynamical Origins of the Little Ice Age and Medieval Climate Anomaly, Science, 326, 1256-1260, 2009.

Marland, G., Boden, T. A., and Andres, R. J.: Global, regional and national emissions, in: Trends: a compendium of data on global change. Carbon Dioxide Information Center, Oak Ridge National Laboratory, US Department of Energy, Oak Ridge, Tenn., USA, 2003.

Marsland, S., Haak, H., Jungclaus, J., Latif, M., and Röske, F.: The Max-Planck-Institute global ocean/sea ice model with orthogonal curvilinear coordinates, Ocean Modell., 5, 91-127, 2003.

Miettinen, A., Divine, D., Ko, N., Godtliebsen, F., and Hall, I. R.: Multicentennial Variability of the Sea Surface Temperature Gradient across the Subpolar North Atlantic over the Last $2.8 \mathrm{kyr}$, J. Climate, 25, 4205-4219, 2012.

Mills, T. C.: Time series modelling of two millennia of northern hemisphere temperatures: long memory or shifting trends?, J. Roy. Stat. Soc. A Sta., 170, 83-94, 2007.

Moberg, A., Sonechkin, D. M., Holmgren, K., Datsenko, N. M., and Karlén, W.: Highly variable Northern Hemisphere temperatures reconstructed from low-and high-resolution proxy data, Nature, 433, 613-617, 2005.
Muscheler, R., Joos, F., Beer, J., Müller, S. A., Vonmoos, M., and Snowball, I.: Solar activity during the last $1000 \mathrm{yr}$ inferred from radionuclide records, Quaternary Sci. Rev., 26, 82-97, 2007.

Pelletier, J. D.: Analysis and Modeling of the Natural Variability of Climate, J. Climate, 10, 1331-1342, 1997.

Peng, C.-K., Buldyrev, S. V., Havlin, S., Simons, M., Stanley, H. E., and Goldberger, A.: Mosaic organization of DNA nucleotides, Phys. Rev. E., 49, 1685-1689, 1994.

Pope, V. D., Gallani, M. L., Rowntree, P. R., and Stratton, R. A.: The impact of new physical parametrizations in the Hadley Centre climate model: HadAM3, Clim. Dynam., 16, 123-146, 2000.

Raddatz, T., Reick, C., Knorr, W., Kattge, J., Roeckner, E., Schnur, R., Schnitzler, K.-G., Wetzel, P., and Jungclaus, J.: Will the tropical land biosphere dominate the climate-carbon cycle feedback during the twenty-first century?, Clim. Dynam., 29, 565-574, 2007.

Reynolds, R. W., Rayner, N. A., Smith, T. M., Stokes, D. C., and Wang, W.: An improved in situ and satellite SST analysis for climate, J. Climate, 15, 1609-1625, 2002.

Roeckner, E., Arpe, K., Bengtsson, L., Christoph, M., Claussen, M., Dümenil, L., Esch, M., Giorgetta, M., Schlese, U., and Schulzweida, U.: The atmospheric general circulation model ECHAM-4: Model description and simulation of present-day climate, MPI Tech. Rep. Rep. 218, 1996.

Roeckner, E., Arpe, K., Bengtsson, L., Christoph, M., Claussen, M., Dümenil, L., Esch, M., Giorgetta, M., Schlese, U., and Schulzweida, U.: The atmospheric general circulation model ECHAM-5, part 1: Model description, MPI Tech. Rep. Rep. 349, 2003.

Rybski, D., Bunde, A., Havlin, S., and von Storch, H.: Long-term persistence in climate and the detection problem, Geophys. Res. Lett., 33, L06718, doi:10.1029/2005GL025591, 2006.

Rybski, D., Bunde, A., and von Storch, H.: Long-term memory in 1000-year simulated temperature records, J. Geophys. Res., 113, D02106, doi:10.1029/2007JD008568, 2008.

Rypdal, K., Østvand, L., and Rypdal, M.: Long-range memory in Earth's surface temperature on time scales from months to centuries, J. Geophys. Res., 118, 7046-7062, 2013.

Rypdal, M. and Rypdal, K.: Long-memory effects in linearresponse models of Earth's temperature and implications for future global warming, J. Climate, submitted, 2013.

Schmidt, G. A., Jungclaus, J. H., Ammann, C. M., Bard, E., Braconnot, P., Crowley, T. J., Delaygue, G., Joos, F., Krivova, N. A., Muscheler, R., Otto-Bliesner, B. L., Pongratz, J., Shindell, D. T., Solanki, S. K., Steinhilber, F., and Vieira, L. E. A.: Climate forcing reconstructions for use in PMIP simulations of the last millennium (v1.0), Geosci. Model Dev., 4, 33-45, doi:10.5194/gmd4-33-2011, 2011.

Smith, T. M. and Reynolds, R. W.: Improved Extended Reconstruction of SST (1854-1997), J. Climate, 17, 2466-2477, doi:10.1175/1520-0442(2004)017<2466:IEROS>2.0.CO;2, 2004.

Smith, T. M. and Reynolds, R. W.: A Global Merged LandAir-Sea Surface Temperature Reconstruction Based on Historical Observations (1880-1997), J. Climate, 18, 2021-2036, doi:10.1175/JCLI3362.1, 2005.

Solanki, S. K., Usoskin, I. G., Kromer, B., Schuessler, M., and Beer, J.: Unusual activity of the Sun during recent decades compared to the previous 11000 years, Nature, 431, 1084-1087, 2004. 
Steinhilber, F., Beer, J., and Frohlich, C.: Total solar irradiance during the Holocene, Geophys. Res. Lett., 36, L19704, doi:10.1029/2009GL040142, 2009.

Syroka, J. and Toumi, R.: Scaling and persistence in observed and modeled surface temperature, Geophys. Res. Lett., 28, 32553258, 2001.

ter Braak, C. J. and Juggins, S.: Weighted averaging partial least squares regression (WA-PLS): an improved method for reconstructing environmental variables from species assemblages, Hydrobiologia, 269, 485-502, 1993.

Usoskin, I. G., Solanki, S. K., and Kovaltsov, G. A.: Grand minima of solar activity during the last millennia, Proceedings of the International Astronomical Union, 7, 372-382, 2011.

von Storch, H., Zorita, E., Jones, J. M., Dimitriev, Y., GonzálezRouco, F., and Tett, S. F. B.: Reconstructing Past Climate from Noisy Data, Science, 306, 679-682, 2004.
Vyushin, D., Zhidkov, I., Havlin, S., Bunde, A., and Brenner, S.: Volcanic forcing improves Atmosphere-Ocean Coupled General Circulation Model scaling performance, Geophys. Res. Lett., 31, L10206, doi:10.1029/2004GL019499, 2004.

Weber, R. O. and Talkner, P.: Spectra and correlations of climate data from days to decades, J. Geophys. Res., 106, 20131-20144, 2001.

Wetzel, P., Maier-Reimer, E., Botzet, M., Jungclaus, J., Keenlyside, N., and Latif, M.: Effects of ocean biology on the penetrative radiation in a coupled climate model, J. Climate, 19, 3973-3987, 2006.

Zorita, E., González-Rouco, F., and Legutke, S.: Testing the Mann et al. (1998) Approach to Paleoclimate Reconstructions in the Context of a 1000-Yr Control Simulation with the ECHO-G Coupled Climate Model, J. Climate, 16, 1378-1390, 2003. 